\title{
Etude sur la déformabilité de la nappe
}

\author{
Pierre-Philip Pechenart ${ }^{1,1}$, Margaux Gillet $^{2}$, et Marc Mimram ${ }^{3}$ \\ ${ }^{1}$ Etudiant à l'ENSAVT, double master Structure \& Architecture avec l'Ecole des Ponts ParisTech \\ ${ }^{2}$ Architecte DE, Ingénieur diplômée de l'Ecole des Ponts Paris Tech, Enseignante à l'ENSAVT \\ ${ }^{3}$ Architecte DPLG, Ingénieur de l'ENPC, Professeur des Ecoles d'architecture
}

\begin{abstract}
Résumé. Cet article vise à explorer la déformabilité d'une nappe plane découpée par un motif. Ce dernier lui donne la capacité de subir des déformations, d'acquérir de la souplesse tout en conservant un continu de matière, et d'évoluer d'une surface plane à une surface complexe -voire à double courbure. Les notions de surface, de surface développable, et de nappe architecturale forment les bases de l'étude du motif et de son fonctionnement. L'étude du fonctionnement d'une poutre entre deux appuis permet de simplifier notre approche, notamment dans l'analyse de ses déformations, de sa résistance au cisaillement, et de l'influence du matériau choisi pour composer cette nappe. Nous verrons dans cet article comment le motif que nous proposons d'étudier permet la double courbure, en quoi il est résistant et souple à la fois, en étudiant notamment les blocages internes de la nappe. La comparaison entre différents motifs nous permettra de mieux le comprendre et l'appréhender. Cette étude participe également au développement d'un pavillon architectural à échelle 1 .
\end{abstract}

Mots-clés. Surface développable, motif, nappe, déformabilité, double courbure, surfaces complexes, souplesse.

\begin{abstract}
This article aims to explore the deformability of a flat net cut by a specific pattern. The pattern gives the net the ability to undergo deformations, to become flexible while keeping a continuum of matter, and to evolve from a flat surface to a complex one - possibly with a double curve. The notions of surface, developable surface, and architectural net form the basis of the pattern's study and its way of functioning. The study of a beam between two supports allows to simplify our approach, in particular for the analysis of its deformations, its resistance to shearing, and the influence of the material chosen to compose this sheet. We will see in this article how the pattern that we want to study allows the double curvature, how it can be resistant and flexible at the same time, by studying in particular the internal blockages of the net. The comparison between different patterns will allow us to better understand and describe it. This study also contributes to the development of an architectural pavilion at a 1:1 scale.
\end{abstract}

Keywords. Developable surface, pattern, net, deformability, double curvature, complex surfaces, flexibility.

\footnotetext{
${ }^{1}$ Auteur principal : pierre-philip.pechenart@hotmail.fr
} 


\section{Introduction}

La construction d'une nappe ou d'une surface ${ }^{2}$ (Pottman et al., 2007, pp. 237-244) à double courbure est un véritable enjeu pour les ingénieurs et les architectes, et peut s'avérer très problématique. En effet, celle-ci requiert une mise en œuvre extrêmement complexe, ce qui pousse à avoir souvent recours à des membranes ou à des tissus déformables et élastiques pour couvrir les surfaces, ou ce qui impose une discrétisation de cette surface en éléments plans de petites dimensions.

La nappe est une définition architecturale et matérielle, c'est-à-dire constructible, de la surface. On peut appliquer à cette nappe une épaisseur, une matérialité, elle peut filtrer la lumière, ou au contraire l'empêcher de se propager.

Dans cet article, nous cherchons à définir une surface complexe à partir d'une surface plane dite développable (Pottman et al., 2007, pp. 531-565), afin d'étudier la déformabilité d'une nappe, c'est-à-dire sa capacité à subir des déformations. Il s'agit principalement d'un travail expérimental, basé sur l'étude progressive de différents motifs répétables et visant à développer un système modulaire intégrant dans sa géométrie un mode d'assemblage. Nous cherchons à expérimenter une nappe composée d'un module souple qui permettrait de déformer la surface, et qu'un assemblage viendrait rigidifier et bloquer.

\section{Démarche et méthodologie}

\subsection{Mise en évidence de la difficulté d'obtenir une double courbure}

Il est quasiment impossible de donner une double courbure à un élément plan continu en lui appliquant des efforts sans mettre en jeu la capacité plastique de cet élément ou sans lui appliquer des efforts importants. Nous faisons le test avec les plaques de carton à notre disposition, dans différentes épaisseurs. Le carton passe en phase plastique, et le matériau se rompt plutôt qu'il ne se déforme en double courbure.

\subsection{Elaboration des modules et assemblages}

Le but de cette recherche est initialement d'élaborer une nappe composée d'un système de modules et d'assemblages dans un même plan, ces derniers permettant de bloquer la nappe dans une certaine position une fois déformée.

Afin de rendre possible la création de courbures à partir d'une surface plane, des incisions sont effectuées dans la surface en suivant la géométrie d'un motif que nous répétons. Cette méthode a l'avantage de garantir le caractère développable de la surface, ce qui la rend facilement réalisable et industrialisable. Pour ce faire, nous procédons à l'inverse du processus de discrétisation d'une surface à double courbure. Dans notre cas, nous partons d'une nappe simple, à savoir une surface développable plane, et nous cherchons quelle(s) déformation(s) nous pouvons lui appliquer, quelle déformabilité elle peut atteindre.

\subsubsection{Essais préliminaires expérimentaux}

Des premiers essais expérimentaux sont menés en décomposant la nappe en modules triangulaires, le triangle étant une forme nous permettant diverses déformations et le module de base le plus couramment utilisé dans les techniques de discrétisation. Ces triangles équilatéraux sont assemblés sur une nappe de tissu (figure 1), et des assemblages équivalents

${ }^{2}$ Mathcurve par R. Férreol, http://www.mathcurve.com/surfaces (consulté le 25/06/2018). 
à des encastrements sont mis en place afin de rigidifier la nappe, notamment certaines arrêtes, afin de fixer les modules entre eux.

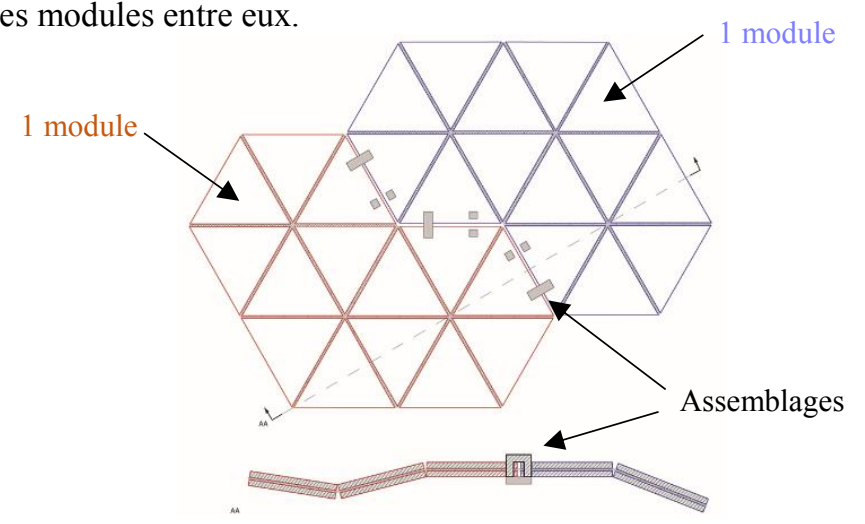

Figure 1. Schéma des triangles assemblés sur le tissu.

\subsubsection{Motifs créés}

Afin de s'approcher de courbures dans la nappe, nous effectuons des découpes de modules serrés sur une feuille de carton de $2 \mathrm{~mm}$ d'épaisseur pour créer une dimension d'éléments évoluant entre eux en se déformant. Ainsi, à la manière de la courbe de Hilbert, un continu de matière le plus constant possible est maintenu afin d'avoir une découpe maximale de la plaque. Quatre motifs ont été ainsi créés et testés au fur et à mesure, évoluant en fonction des résultats obtenus : le $1^{\mathrm{er}}$ motif est dessiné de manière à obtenir un modèle à une direction (forme de peigne), le $2^{\mathrm{e}}$ est formé sur une base carrée et les deux derniers à partir d'une base hexagonale (figure 2). Le logiciel AutoCad a été utilisé pour dessiner les motifs, qui ont ensuite été usinés à l'aide d'une machine de découpe laser.

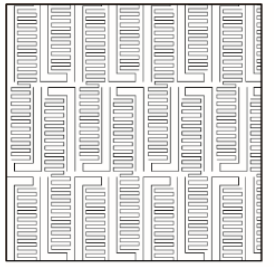

$1^{\text {er }}$ motif - Peigne

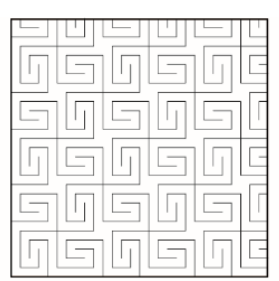

$2^{\text {ème }}$ motif - Base carrée

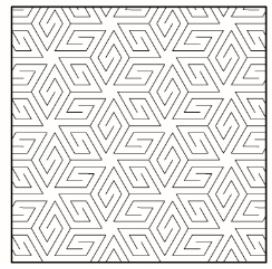

$3^{\text {ème }}$ motif - Base hexagonale 1



$4^{\text {ème }}$ motif - Base hexagonale 2

Figure 2. Schéma des quatre motifs créés.

\subsection{Fonctionnement du motif et choix du matériau}

La caractéristique qui nous intéresse plus particulièrement pour comprendre le fonctionnement du motif est la résistance au cisaillement dans le plan de la nappe.

Sous l'action des forces de cisaillement, la plaque tend à se séparer en deux morceaux glissant l'un par rapport à l'autre dans le plan de section droite ${ }^{3}$. C'est ce phénomène de

\footnotetext{
${ }^{3}$ Sollicitation de cisaillement, résistance des matériaux cisaillement, par H. Jardin-Nicolas, http://herve.jardin-nicolas.pagesperso-orange.fr/TSTI/MECA $\% 20 \mathrm{fevr} /$ cisaillement (consulté le 30/06/2018).
} 
cisaillement qui apparait lorsque l'on fait les incisions selon le motif dans la plaque, et les déplacements engendrés entre les éléments permettent la souplesse au sein de la nappe.

Par ailleurs, le type de matériau influe également sur les propriétés d'une nappe (Badel, 2011 ; Douthe et al., 2017). Il faut donc être vigilant et prendre en compte le matériau lors de l'étude du motif. Le choix du medium ou du carton nous parait être ici le plus propice pour une approche empirique du fonctionnement de la nappe, car étant des matériaux reconstitués, ce sont des matériaux isotropes. Seule la découpe dans cette nappe influera sur la souplesse de celle-ci ${ }^{4}$.

\section{Résultats}

\subsection{Essais préliminaires expérimentaux}

La nappe créée avec les triangles équilatéraux comporte des arrêtes rigides grâce aux assemblages, et reste plane lorsqu'on ne la sollicite pas. Cependant, il est possible de manipuler les triangles dans plusieurs positions au sein d'un même module, et donc de créer des courbures, cela grâce à l'élasticité du tissu associée à la forme triangulaire. En plaçant des pyramides en dessous de la nappe, on parvient à créer un système en 3 dimensions en approximant des courbures. Posées aléatoirement et se bloquant par frottement, les pyramides figent la nappe, en fonction de leur emplacement et de leur angle (figure 3).



Figure 3. Nappe de modules triangulaires en 3 dimensions.

Cependant, une double courbure ne peut pas être atteinte avec ce modèle, les assemblages rigides entre les modules permettant de former seulement des simples courbures. De plus, du fait de la forme des modules et des assemblages, cette nappe a très peu de possibilités pour se déformer, c'est pourquoi une alternative au module a été cherchée. Ce schéma n'est en effet pas optimal car le but de cette étude est entre autres d'avoir la plus grande déformation possible afin de former différentes courbures.

\subsection{Motif à une direction}

Cette première découpe en forme de peigne a permis de créer un réseau d'éléments linéaires plutôt qu'une plaque, de libérer des degrés de liberté au sein de la nappe et donc d'induire de la souplesse dans celle-ci (figure 4). Cependant, selon le sens des découpes, on constate qu'une direction de la nappe est très souple tandis que l'autre ne l'est pas du tout.

Ici aussi, un système pour bloquer la nappe dans une certaine position a été envisagé, au travers de L découpés dans le carton et intégrés au motif. Cependant, les résultats ne se sont pas avérés satisfaisants, car les contraintes dans les L étaient trop importantes et ces assemblages étaient très difficiles à mettre en œuvre. Il a donc été décidé à ce stade de l'étude

\footnotetext{
${ }^{4}$ http://webentwood.com/portfolio/developpabilite (consulté le 25/06/2018).
} 
d'abandonner la volonté de figer la nappe dans l'espace et de la contraindre dans une seule position seulement. En effet, chercher à rendre la nappe autobloquante est aussi très contraignant vis-à-vis de l'élaboration et du design du motif, qui doit prendre en compte les assemblages. Cela est donc un frein à l'étude de la déformabilité de la nappe et de l'obtention de double courbure à partir d'une surface développable.
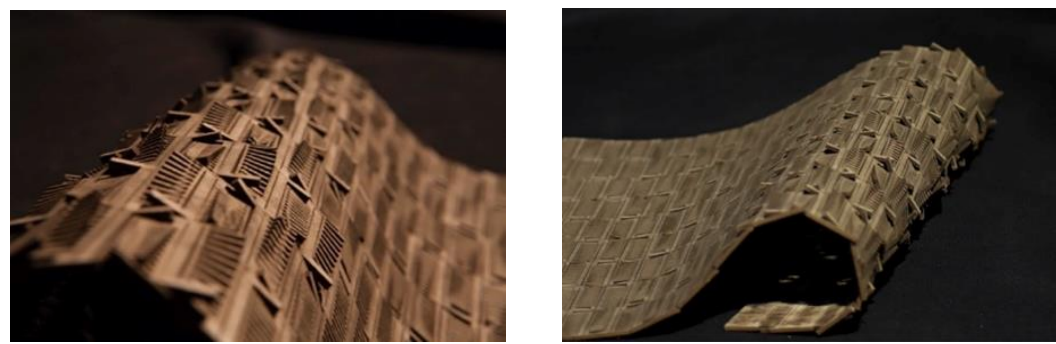

Figure 4. Motif en forme de peigne, extrêmement déformable dans une direction.

Le but est donc maintenant d'approfondir le plus possible la recherche de déformation au sein de la nappe et la création de courbures, en laissant libre le choix des courbures et la position de la nappe une fois mise en forme.

\subsection{Motif à base carrée}

Une meilleure déformation du carton est constatée avec le motif à base carrée (figure 5), car cette fois-ci deux directions sont privilégiées et permettent de libérer des degrés de liberté au sein de la nappe. Cependant, la double courbure est toujours impossible à atteindre avec ce motif, hormis très légèrement aux nœuds de transition entre deux courbures, car trop de directions sont encore bloquées dans la nappe, notamment les directions diagonales par rapport aux axes du carré.

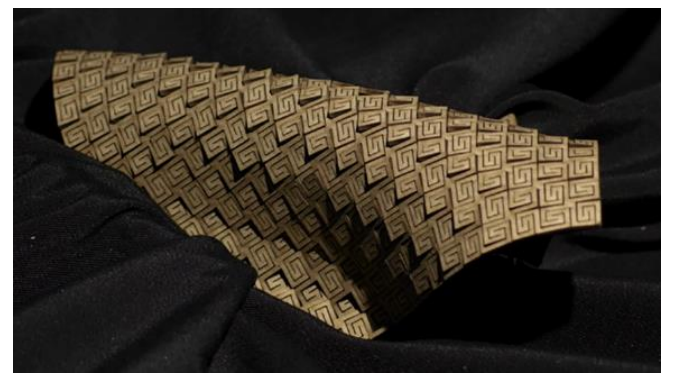

Figure 5. Motif à base carrée, déformable dans plus de directions que le peigne.

\subsection{Motif à base hexagonale}

Un premier motif à base hexagonale a alors été créé, en espérant que l'augmentation du nombre de côtés permette de libérer plus de directions et donc plus de souplesse (figure 6a). Cependant, on constate que cette nappe est encore moins souple que celle à base carrée, car le motif a été dessiné de manière à ce que six branches différentes du motif se rejoignent en un point solide de la nappe (contrairement à quatre pour le carré). Cela rigidifie donc la nappe au lieu de la rendre plus déformable. 

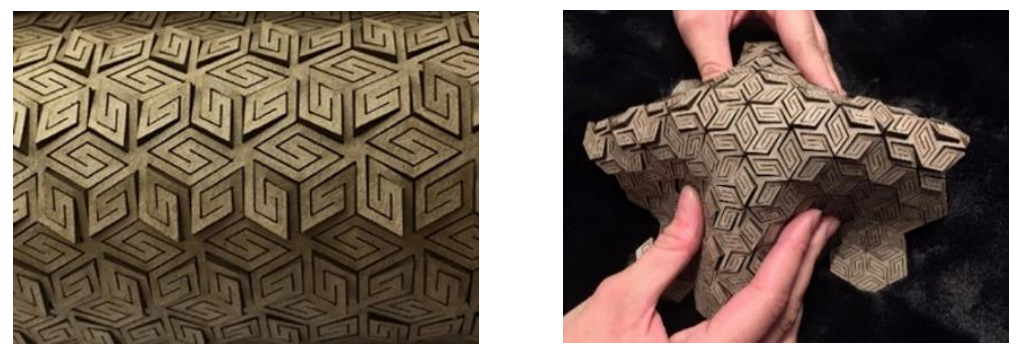

Figure 6. (a) Motif à base hexagonale 1, (b) et à base hexagonale 2 .

Un second motif hexagonal a donc été dessiné en "inversant» le précédent, afin d'effectivement libérer des nouvelles directions. Dans ce cas, on observe que la nappe est très souple, qu'elle peut subir de grandes déformations, et qu'une double courbure est possible avec ce modèle (figure $6 \mathrm{~b}$ ). Cela est dû au fait que, dans cette version du motif, seules trois branches se relient en un point, ce qui permet de libérer six extrémités d'hexagones, contre trois pour le premier (figure 7).
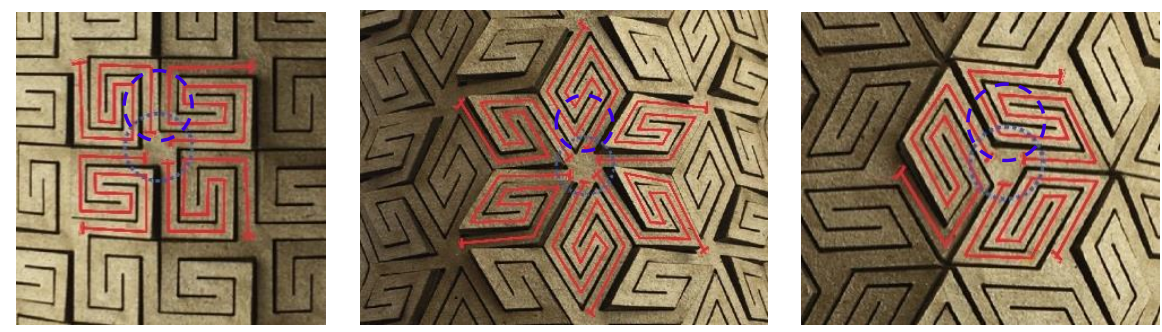

Figure 7. Nombre de branches reliées aux nœuds des motifs à base (a) carrée, (b) hexagonale 1, (c) hexagonale 2.

De plus, il privilégie plus de directions que les motifs carrés et en forme de peigne, ce qui en fait le motif le plus souple et le plus intéressant à retenir (figure 8).

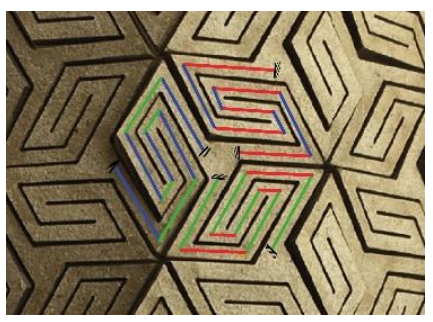

Figure 8. Le motif à base hexagonale 2 favorise trois directions différentes.

La nappe semble fonctionner comme une rotule hors de son plan et comme un encastrement dans son plan. Le motif choisi apparait comme une poutre encastrée qui s'enroule sur elle-même et qui a donc une grande longueur sans pour autant occuper beaucoup d'espace (figure 9). On a optimisé la largeur de la poutre afin qu'elle mesure $2 \mathrm{~mm}$, ce qui correspond à l'épaisseur de la plaque de carton. Ce qui nous permet également de pouvoir la solliciter selon l'axe $\mathrm{z}$ autant que selon les axes $\mathrm{x}$ et $\mathrm{y}$. 


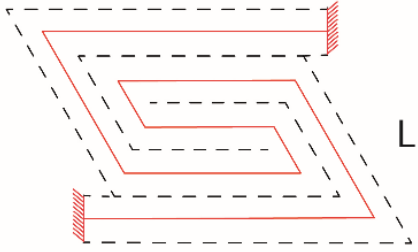

Axe de la poutre



$\mathrm{L}$

Figure 9. Ce profil de poutre enroulée permet de gagner en souplesse en diminuant l'impact de la longueur de la poutre.

Le motif en forme de peigne, en comparaison, favorise grandement une direction plutôt que l'autre, car la longueur de la poutre s'étend dans une direction seulement (figure 10). La largeur de cette poutre a été réduite à $1 \mathrm{~mm}$ afin de laisser davantage de souplesse dans la direction privilégiée, mais la nappe devient alors beaucoup plus cassante dans les autres directions.

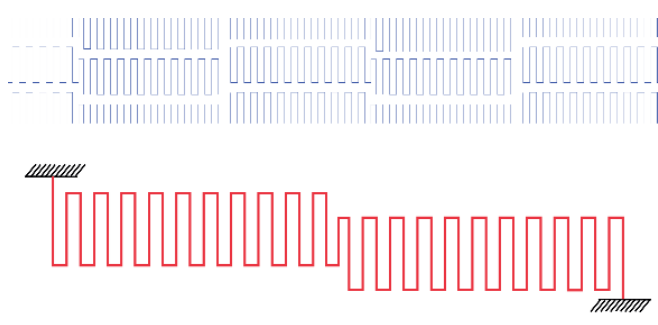

Figure 10. Le motif en forme de peigne favorise une direction par rapport aux autres.

\subsection{Approche mathématique}

Afin de comprendre le fonctionnement de ces découpes sur la nappe, on apparente les motifs à un entrelacement de poutres encastrées. Par simplification, on étudie la nappe comme une poutre isostatique entre deux appuis, la poutre ayant une inertie de la largeur de la plaque. Si on divise cette plaque en deux, on obtient alors deux poutres sur deux appuis chacune et une inertie diminuée de moitié. Et ainsi de suite. Cette méthode sert à approximer une surface complexe afin de la décomposer en surfaces plus simples (Laurent, 2012 ; Lee et al., 1999), et nous essayons ici de parcourir le chemin inverse.

Notre motif est donc comparable à une succession de poutres isostatiques sur appuis soumises à une charge ponctuelle ou à une charge répartie.


Figure 11. Schéma statique d'une poutre sur deux appuis, sollicitée par (a) une force ponctuelle $P$, (b) une force répartie $q$. 
Lorsqu'une charge ponctuelle $P$ est appliquée au milieu d'une poutre entre deux appuis (figure 11a), le moment maximum dans la poutre sur deux appuis, qui dépend de sa géométrie, se trouve au milieu de la poutre et est donné par :

$$
M_{L / 2}=P L / 4
$$

Avec :

$P$ : la force appliquée

$L$ : la longueur de la poutre

Cela signifie que si l'on veut que la poutre se déforme et soit de plus en plus souple, on peut augmenter la force $P$, mais la déformation sera alors dans le matériau lui-même qui passe en phase plastique, et non plus dans sa géométrie, et la poutre va finir par rompre. Il est donc impossible d'utiliser cette variable pour induire des courbures dans la plaque.

C'est pour cela que l'on a joué sur le second paramètre, à savoir la longueur de la poutre, pour augmenter la souplesse de la nappe et créer des déformations.

Par ailleurs, la flèche maximale d'une poutre sur deux appuis se situe à la moitié de la longueur maximale et s'écrit telle que :

$$
F=P L^{3} /(48 E I)
$$

Avec :

$E$ : module d'Young

$I$ : inertie de la poutre

La longueur de la poutre est élevée au cube, ce qui en fait la variable la plus importante dans la déformation de la poutre.

$\mathrm{Ne}$ voulant faire varier ni l'inertie de la nappe ni le matériau la composant (donc en conservant le même module d'Young), ce paramètre est donc le seul nous permettant de modifier la structure de la nappe. C'est pourquoi nous avons fait varier les motifs de découpe de la plaque, en essayant d'allonger au maximum la longueur des «poutres » du motif afin d'avoir la plus grande déformabilité possible.

Le même raisonnement est valable lorsqu'on applique une charge répartie q (figure 11b) sur une poutre simple entre deux appuis. Cette modélisation nous permet donc de théoriser et justifier le développement des différents motifs et leur évolution.

\section{Discussion et analyse}

La double courbure dans une nappe est, comme dit précédemment, un véritable challenge. En effet, il faut réussir à courber dans deux directions différentes une plaque qui possède une certaine rigidité. La plaque doit rester souple tout en conservant de la résistance dans son plan, c'est-à-dire qu'il faut combiner la rigidité naturelle du matériau avec la souplesse de la géométrie dessinée.

En structure, on distingue plusieurs types de blocage possibles, qui s'appliquent pour les conditions limites d'une structure, mais également au sein même de la structure : ce sont les degrés de blocage interne de la nappe. C'est sur ces blocages internes que nous jouons pour obtenir une double courbure dans notre nappe. Nous distinguons deux types de nœuds au sein du motif retenu. Le premier joue le rôle d'encastrement et consiste en un assemblage de 3 barres encastrées. Ce nœud permet de gagner ou de conserver une résistance dans le plan et même hors du plan, ce qui permet à la nappe de rester solidaire. La matière se propage en serpentant à travers la nappe, ce qui lui permet de reprendre les efforts successivement normaux et tranchants soumis dans le plan. En manipulant cette dernière en traction, les modules se déforment en plan et hors-plan, ce qui peut générer de la double-courbure. 
L'autre nœud, quant à lui, est formé de six entailles. C'est le croisement des six barres qui concordent en un point (figure 12a). Ce point n'est pas fixe, c'est un bord libre, qui permet toute la souplesse de la nappe. C'est également en ce sommet que, mise en forme, la nappe va avoir une double courbure. En effet, retenues par les encastrements, les barres vont rester solidaires à des parties différentes de la nappe et donc le nœud libre aura tendance à se relâcher, c'est-à-dire que le point concourant des barres se divisera en six points distincts (figure 12b).
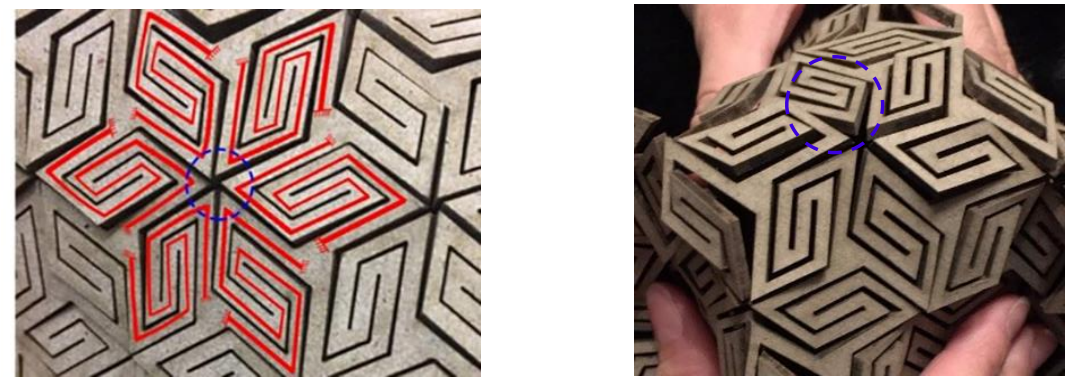

Figure 12. (a) Au repos, les branches se rejoignent pour former un bord libre, (b) puis en déformant la nappe, les points s'éloignent pour créer des courbures.

On comprend ainsi les résultats obtenus quant à la souplesse des différents motifs :

Tableau 1. Caractéristiques des différents motifs

\begin{tabular}{|c|c|c|}
\hline Motif & $\begin{array}{c}\text { Nombre de branches } \\
\text { encastrées }\end{array}$ & $\begin{array}{c}\text { Nombre de bords } \\
\text { libres }\end{array}$ \\
\hline Motif à base hexagonale 1 & 6 & 3 \\
\hline Motif à base carrée & 4 & 4 \\
\hline $\begin{array}{c}\text { Motif à base hexagonale 2 } \\
\text { (final) }\end{array}$ & 3 & 6 \\
\hline Motif à une direction (peigne) & 2 & $\begin{array}{c}\text { Sur toute la longueur } \\
\text { du peigne }\end{array}$ \\
\hline
\end{tabular}

Le motif à base hexagonale 1 est bien le moins souple car il possède un très grand nombre d'encastrements, ce qui rigidifie la structure. La nappe carrée est un peu plus souple car elle possède moins d'encastrements et plus de bords libres, et le motif hexagonal 2 retenu dans cette étude donne le meilleur résultat car il possède seulement 3 encastrements, ce qui permet aussi de libérer plus de directions donc plus de choix dans les courbures, et d'obtenir des doubles courbures. Le peigne quant à lui, est un motif plus particulier car il possède seulement 2 encastrements et des bords libres sont présents sur toute la longueur du peigne, ce qui le rend extrêmement souple dans l'axe perpendiculaire au peigne seulement. En revanche, dans les autres directions, il reste très rigide du fait de l'encastrement trop important entre les différentes rangées de peignes.

\section{Conclusion}

Cette étude vise à comprendre et à appréhender la déformabilité d'une nappe et son fonctionnement. L'objet créé a été obtenu de façon empirique, en testant différents motifs et en essayant d'obtenir la plus grande déformation de la plaque et la possibilité de faire le plus de courbures possibles au sein de la nappe. L'étude des différents motifs passe alors par l'étude du fonctionnement d'une poutre entre deux appuis, qui s'apparente à l'organisation locale de la nappe. 
La nappe ainsi réalisée sur une base hexagonale a de nombreuses propriétés, à savoir une souplesse mêlée à sa résistance, une capacité de développement et de déformation, etc. D'autres essais pourraient être menés sur des pavages archimédiens ou platoniciens afin de voir s'il serait possible d'améliorer encore plus la déformabilité de la nappe en associant différents types de modules.

Par la suite, cette étude a été sélectionnée pour donner lieu à un travail de groupe, sous la forme d'un pavillon à construire, c'est-à-dire d'une nappe à échelle 1 où nous pourrions alors appliquer les raisonnements de cet article (figure 13).

Le bois, à savoir du contreplaqué bouleau à plis mince, a été retenu pour créer les modules de la nappe, pour ses facilités d'usinage, sa bonne résistance, et pour la superposition des très fines couches de bois dont les fibres sont orientées différemment et permettent de n'avoir aucune zone défavorable dans le motif. L'encastrement des trois branches du motif a été réalisé avec des platines en acier boulonnées à 6 endroits ( 2 par branche), ce qui garantit un véritable encastrement. Au total, pour une nappe de $40 \mathrm{~m}^{2}, 110$ plaques de deux hexagones ont été usinées et assemblées. Une fois les hexagones assemblés, les câbles ont été serrés et passés au centre de certaines platines, choisies pour que chaque point reprenne le même poids. Des essais de robotisation ont ensuite été effectués grâce à des moteurs reliés à des interfaces Arduino, afin que la nappe puisse rester en mouvement et créer des jeux de lumière et des atmosphères différentes.

La création d'un pavillon à partir de la déformation d'une nappe a donc été un challenge très intéressant, et la recherche de réponses pour la mise en œuvre d'une surface complexe par exemple en double courbure - reste un sujet passionnant.

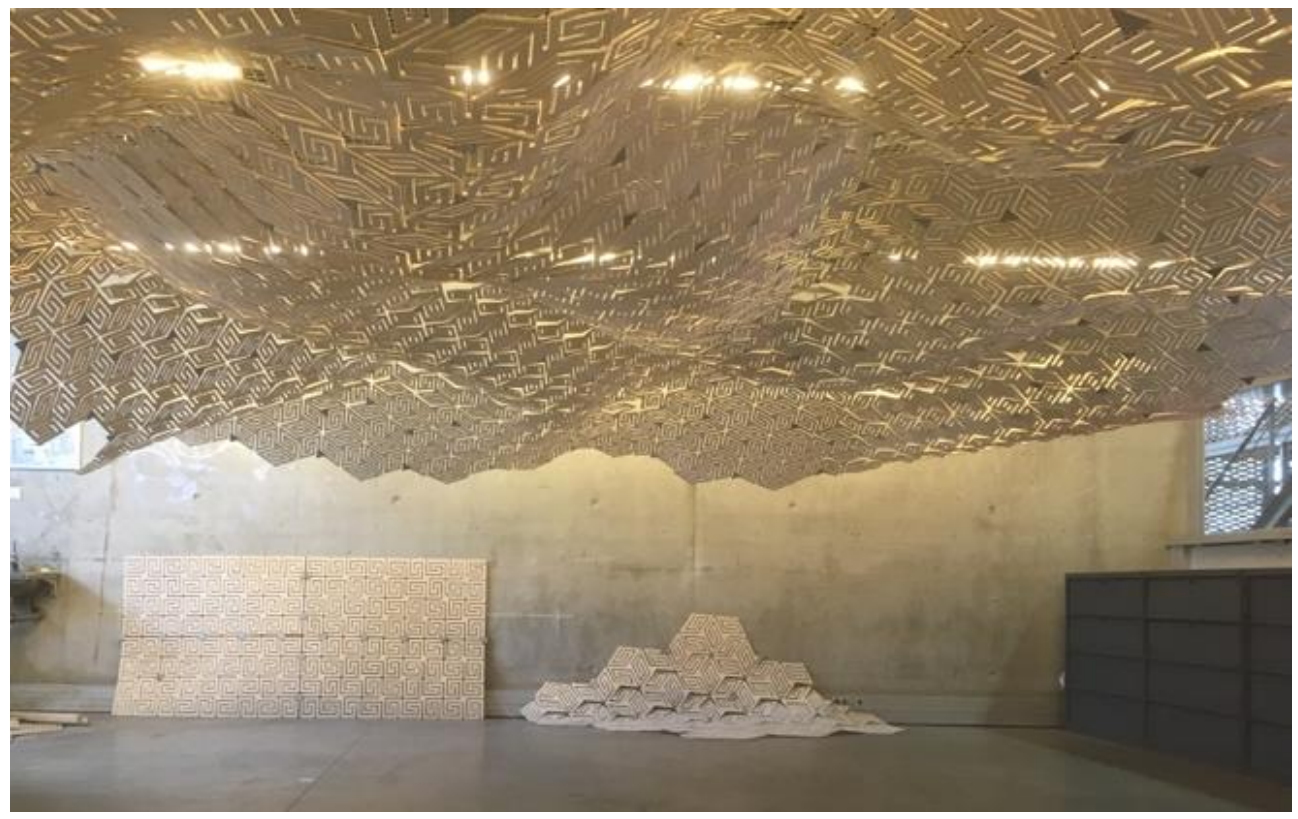

Figure 13. Pavillon à échelle 1, formé à partir de la nappe à motif hexagonal.

\section{Bibliographie}

Badel, P. (2011). Cycle Préparatoire Médecin-Ingénieur 2011-2012. Cours de résistance des matériaux. Ecole des Mines de Saint-Etienne. https://www.emse.fr/ badel/12-0106 COURS RDM FULL.pdf 
Chen, H.-Y., Lee, I.-K., Leopoldseder, S., Pottmann, H., Randrup, T. and Wallner, J. (1999) On Surface Approximation Using Developable Surfaces. Graphical Models and Image Processing, 61(2), 110-124. doi: 10.1006/gmip.1999.0487.

Douthe, C., Du Peloux, L., \& Mesnil, R. (2017). Construire le courbe - O3ATC. Ecole des Ponts ParisTech.

Laurent, G. (2012). Approximation de surfaces développables pour la modélisation d'objets 3D. https://ensiwiki.ensimag.fr/images/c/c3/Irl rapport gilles laurent.pdf

Pottmann, H., Asperl, A., Hofer, M., \& Kilian, A. (2007). Architectural Geometry. Bentley Institute Press. 